\title{
Enacting simulation: A sociomaterial perspective on students' interprofessional collaboration
}

Sofia Nyström, Johanna Dahlberg, Håkan Hult and Madeleine Abrandt Dahlgren

\author{
Linköping University Post Print
}

\section{Tweet}

N.B.: When citing this work, cite the original article.

This is an electronic version of an article published in:

Sofia Nyström, Johanna Dahlberg, Håkan Hult and Madeleine Abrandt Dahlgren, Enacting simulation: A sociomaterial perspective on students' interprofessional collaboration, 2016, Journal of Interprofessional Care,

Journal of Interprofessional Care is available online at informaworldTM:

http://dx.doi.org/10.3109/13561820.2016.1152234

Copyright: Taylor \& Francis: STM, Behavioural Science and Public Health Titles

http://www.tandf.co.uk/journals/default.asp

Postprint available at: Linköping University Electronic Press

http://urn.kb.se/resolve?urn=urn:nbn:se:liu:diva-128254 


\title{
Enacting simulation:
}

\section{A sociomaterial perspective on students' interprofessional collaboration}

\author{
Sofia Nyström*, Johanna Dahlberg**, Håkan Hult**, Madeleine Abrandt Dahlgren** \\ * Department of Behaviour Sciences and Learning, Linköping University, Sweden \\ ** Department of Medicine and Health, Linköping University, Sweden
}

\begin{abstract}
Full-scale simulation exercises are becoming more common as an educational feature of the undergraduate training of health professionals. This study explores how interprofessional collaboration is enacted by the participating students. Practice theory is used as the theoretical framework for a field study of two naturalistic educational settings, when medical and nursing students come together to practice in a simulated emergency situation, where a manikin is replacing the patient. Eighteen sessions of simulations were observed, and data were collected through standardised video recordings that were analysed collaboratively. To ensure transparency and scientific rigour, a stepwise constant comparative analysis was conducted, in which individual observations within and across single video recordings were compared, negotiated and eventually merged.
\end{abstract} The findings show that the student teams relate to the manikin as a technical, medical and human body, and that interprofessional knowings and enactments emerge as a fluid movement between bodily positioning in synchrony and bodily positioning out of synchrony in relation to the sociomaterial arrangements. The findings are related to contemporary theorisations of practice comprising an integrated view of body and mind, and it is discussed how the findings can be used in simulation exercises to support participants' learning in new ways.

Keywords: Simulation education, interprofessional collaboration, health care, learning, practice theory, qualitative video analysis 
* Corresponding author:

Sofia Nyström

Department of Behaviour Sciences and Learning

Linköping University

SE-58183 Linköping

Sweden

Telephone: +461328 2095

Fax: + 4613282145

E-Mail: sofia.nystrom@liu.se 


\title{
Enacting simulation: A sociomaterial perspective on students' interprofessional collaboration
}

\begin{abstract}
Full-scale simulation exercises are becoming more common as an educational feature of the undergraduate training of health professionals. This study explores how interprofessional collaboration is enacted by the participating students. Practice theory is used as the theoretical framework for a field study of two naturalistic educational settings, when medical and nursing students come together to practice in a simulated emergency situation, where a manikin is replacing the patient. Eighteen sessions of simulations were observed, and data were collected through standardised video recordings that were analysed collaboratively. To ensure transparency and scientific rigour, a stepwise constant comparative analysis was conducted, in which individual observations within and across single video recordings were compared, negotiated and eventually merged. The findings show that the student teams relate to the manikin as a technical, medical and human body, and that interprofessional knowings and enactments emerge as a fluid movement between bodily positioning in synchrony and bodily positioning out of synchrony in relation to the sociomaterial arrangements. The findings are related to contemporary theorisations of practice comprising an integrated view of body and mind, and it is discussed how the findings can be used in simulation exercises to support participants' learning in new ways.
\end{abstract}

Keywords: Simulation education, interprofessional collaboration, health care, learning, practice theory, qualitative video analysis 


\section{Introduction}

Health care services and professionals around the world are under increasing pressure coping with diminishing resources, and the simultaneous demands to improve quality in practice and enhance patient safety. These changing conditions present knowledge and learning challenges in ongoing work, and have implications for the education and continuing development of health professionals. Interprofessional collaboration and teamwork has been emphasised as one of the means, but also challenges in order to accomplish a sustainable and safe future health care, requiring also a renewal of professional health care education (WHO 2010, Frenk et al 2010). Simulation of health care work, where a technologically advanced manikin replaces the patient, is increasingly being used in undergraduate programmes as a complement to clinical placements as well as a way to arrange a safe environment for training of clinical and interprofessional skills for professional practice in health (Cant \& Cooper, 2010). Against the backdrop of the increasing use of simulation as a means of professional and interprofessional learning, the need to understand how to arrange these activities, in order to best support students' learning, becomes an important research issue. This paper reports findings from a nationally funded research project on simulation-based education in undergraduate health care programmes in collaboration between three universities in Sweden. Reviews of research on interprofessional simulation-based training show that studies focusing on the practice of simulation in undergraduate programs are still less common (Gough, Hellaby, Jones \& MacKinnon, 2012; Palaganas, Epps, \& Reamer, 2014). Instead, research in interprofessional education (IPE) and simulation in undergraduate training have predominantly concerned evaluation of courses, learners’ perspective of IPE, and teamwork outcomes (Cook et al., 2011). Some studies also focused on the learners' attitudes and 
their learning about the roles and skills of other professions or disciplines (Gough et al., 2012; Alinier, Harwood, Harwood, Montague, Huish \& Ruparelia, 2014).

The research on simulation has also been suggested as too evaluative and protocol-driven, neglecting theoretical groundwork (Berragan, 2011). Dieckmann et al. (2012) propose that there is a need for more theorised, process-oriented analyses of current simulation practice. This study will contribute to the knowledge of simulation by focusing on the situated practice and arrangements of simulation-based education, i.e. what actually takes place as medical and nursing students collaborate in a simulated setting. By doing so, the study respond to the call for more theoretically framed research in simulation. The focus on practice also leads to the choice of a practice theory approach (Schatzki, 2002) as the framework and perspective for the study, as will be described in the following section.

\section{Theoretical perspective}

Hager et al. (2012) argue that discourses of the nature of professional knowledge and learning at large are changing (Hager et al., 2012). The dominating scientific, technical rationalities of professional practice as simply the application of theoretical knowledge, possessed by individuals, are being challenged. Recent theorisations of practice (Schatzki, 2002; Gherardi, 2009; Green \& Hopwood, 2015; Kemmis, 2009) instead suggest alternative views of knowledge as being embodied and relational, intertwined with ethical reasoning and materiality. Practices evolve in historical and various social contexts. Professional learning and knowing-in-practice is thereby seen as embedded in practical rationalities, emergent and enmeshed with social and material arrangements (Schatzki, 2002; Gherardi 2009; Kemmis 2009; Fenwick \& Abrandt Dahlgren, 2015). 
In Schatzki's (2002) terms, a practice is an organised nexus of action, a set of bodily doings and sayings. These bodily doings and sayings are organised by a practical understanding of the knowing in a practice. The practical understanding and performance of practice is influenced by rules and, what Schatzki labels, teleoaffective structures. Rules are explicit normative enjoinings that regulate the actions, while the teleoaffective structures guide the activities in the practice towards a range of acceptable actions and acceptable ends. Furthermore, the performance is influenced by a general understanding, referring to a broader understanding of the practice as a whole.

Pedagogically, a cycle of simulation typically follows three phases; briefing, simulation and debriefing. These phases encompass different challenges to educators and learners and have different sociomaterial arrangements and activities (Ahn et al., 2015). The present paper has a particular focus on the simulation phase, and the aim of the study is to describe and analyse how interprofessional collaboration emerges in a simulated emergency situation as enacted by participating students.

\section{Methodology}

In order to resonate with the theoretical perspective on simulation as a practice, the study was conducted through observations of simulations in their naturalistic settings. The appropriateness of employing ethnographic methods, in alignment with a sociomaterial perspective, has been emphasised, since ethnography has a long tradition of focusing on material cultures (Fenwick et al., 2011, Schatzki, 2012). Ethnography does not have a univocal definition, but is used in different ways on different occasions (Hammersley, 2006). Hammersley, however, identifies an important feature common to ethnographic 
studies, namely to “...study at first hand what people do and say in particular contexts.” (p. 4). In doing so, the methods applied are often involving participant observations in relevant settings, interviews and documents. The contexts in this study are two sites (site 1 and site 2) of undergraduate education of health professionals, which are relevant for the study since they include simulation as part of their curricula. The sample comprised students participating in a full day of simulation exercises. As part of the exercise, students were grouped into interprofessional teams that were kept together during the simulation day. At both sites, the interprofessional simulation was a compulsory part in the last semester before graduation. All scenarios included were variations on the themes of acute emergency and/or deteriorating conditions of the patient. In site 1 the simulations took place in a fully equipped emergency room, operated from the control room by the instructor and a technician with a oneway window, giving a view of the simulation room. In site 2 the scenario was played out in a health care centre. In common, the simulator was a human-sized manikin (site 1 used a Laerdal SimMAN 2 and site 2 used a Laerdal SimMAN 3G).

\section{Participants}

All students in the medical and nursing programmes involved in the simulation day at the two sites were invited to participate in the study. In total, 106 students - 71 females and 35 males accepted to participate in the session to be video recorded. Sixty-six were nursing students and 40 were medical students. Some students had prior experience of interprofessional simulation with students from other programmes, although a majority had only experienced skills training within their own professional programme.

\section{Data collection}


As a means of data collection, all simulation sessions were video recorded. The strength of the usage of video is that it creates a unique opportunity for conducting an analysis of the students' actions and social interactions, and how these are entangled with material arrangements (Hopwood, 2013). The video recordings also allow for repeated observation and field note taking. According to Schatzki (2002), the social and material arrangements prefigure practical intelligibility, i.e. certain activities become more likely to happen. At the same time, video recordings are delimited to what can be captured through observation, which leaves the researchers' interpretation of practical and general understandings, rules and teleoaffective structures in use, to what can be reflected in the observed actions. Altogether, two video cameras were placed in different positions in order to capture all actions of the participants in the simulation room.

\section{Data analysis}

The empirical data analysed in this paper is based on 18 hours of video recordings. A purposeful approach to collaborative data analysis, comprising a layered process in three phases of activities, was developed and utilised by a group of four researchers (see details in table 1). The process was inspired by constant comparative analysis as described by Boeije (2002) and video analysis as described by Heath, Hindmarsh, and Luff (2010). Table 1 illustrates the different phases of the collaborative analysis. As a first phase, individual field notes were taken while observing the video recording together. The theoretical perspective directed the analytical attention towards how the nexus of actions hanged together as relationships between material entities including both human actors, such as bodies, and non-human actors, such as the arrangements of patients' and students' bodies, clinical environments, and protocols in use. The individual field notes were cross-checked, compared and finally merged and transcribed into a 
joint, preliminary description of the enacted interprofessional collaboration as a relationship between bodily movements and arrangements. This first phase made it possible to obtain a shared understanding, as well as to articulate the focus for the second phase, in which comparisons between different video-recordings of the same scenario were made. The second phase allowed us to identify the preliminary findings regarding bodily movements and sociomaterial arrangements across different video-recordings, and also made it possible to discern a variation in students' attunement to the patients' body, and regards patterns of synchrony in relation to sociomaterial arrangements. In the third phase, comparisons were made between video recordings of different scenarios, with the purpose of enlarging and refining the provisional interpretation against all data. The third phase resulted in the identification of two patterns of enacting interprofessional collaboration, described in relationship to sociomaterial arrangements.

(Insert Table 1 here)

\section{Ethical considerations}

In accordance with ethical research practices, the participants, i.e. the students, instructors and operators, provided their consent to participate after they had been informed of the studys' aim and of how the research material would be used. All students participated in the simulation exercise as part of their course, but only those who had given their consent to participate were video recorded. The research project was ethically approved by Linköping University (Dnr 2012/439-31). 


\section{Findings}

In the analysis of how students' actions and interactions were entangled with social and material arrangements, it was apparent that students were juggling different enactments of the patients' body. They related to the manikin as a technical body, as they performed assessments in a way that the technical features of the manikin allowed. Secondly, they related to the manikin as a physical/medical body, as they performed their medical knowing in the situation, following the ATLS protocols. Finally, they related to the manikin as a human body, caring for the manikin like a real person, in their suspension of disbelief, for example by tucking in a blanket around the feet in order to keep them warm. The aim of the simulation exercise was to provide opportunities for the students to practice professional skills as well as teamwork and collaboration. The findings showed how interprofessional knowings and enactments are emerging as a fluid movement between bodily positionings in synchrony and bodily positioning out of synchrony with the sociomaterial arrangements.

Bodily positionings in synchrony

When bodily positionings were in synchrony with the sociomaterial arrangements in interprofessional collaboration, the movements of the student team members were connected in a fluid chain of actions. The chain of action was composed of different doings in interaction between the students. The activities of the chain comprised noticing a sign indicating a deterioration of the patients' condition (auditory or visual), which was followed by enactment of leadership through taking action, or responding through attuning to the action of others. In the attunement to the action of others, there was also anticipation of the next action in the way the material arrangements were related to. This chain of actions in interprofessional collaboration is illustrated below by a transcribed video sequence of a critical incident in scenario where the patient, who has a head trauma after a car accident, starts to vomit. 
The medical student in charge of the patient directly steps up and secures the head and says, "We need to turn Sofia. Position yourselves!”. The second medical student and one of the nursing students move to the same side of the patient, secure the arm with the catheter and prepare to turn the patient. The medical student at the head then says "On the count of three! One, two, three". Simultaneously they turn Sofia and the second nurse puts a bowl in front of Sofia's mouth. The second medical student moves to the head and secures the airways. Once the patient is stabilized, still on her side, the second medical student moves again to investigate the back and spine for injuries, after which the patient is turned back to the original position. (Site 1 simulation 3)

The pattern of the collaboration is illustrated graphically in figure 1. (insert figure 1 here)

Another example of how leadership is enacted in synchrony with sociomaterial arrangements is when a nurse student, in the very beginning of a scenario, starts washing her hands, and hands out aprons and gloves to the rest of the students (Site 1- simulation 1). This makes the other students react and they all become active in getting prepared, discussing how they should work and collaborate.

The two examples show how sayings and doings, as well as bodily movements are connected to materiality. The nursing and the medical students demonstrate their respective professional 
knowings in their enactments of the manikin as a medical and human body, but through the synchronisation of bodily positions, their sayings and doings in the critical situation also enact interprofessional knowing. According to Schatzki (2002), material arrangements prefigure the emerging practice. The material arrangements, for example in the emergency room, make some actions easier to take or follow than others. When the students immerse themselves in the learning experience and accept the limitations of the simulation, disbelief is fully suspended. Our data show that even though the material equipment was not even present, it still prefigured the actions taken, defining the 'as if' simulation situation as a de facto 'as is' situation. The material arrangements and the constraints of the simulation contributed to students juggling with the different enactments of the patients' body. During one simulation (Site 1- simulation 4) the patient stopped breathing and all students dropped everything they were doing and focused on saving the patient, i.e. the human body. In the speakers you then heard the instructor saying, "The patient is breathing, so continue as first planned". This example shows how the technical breakdown of the simulator, in this case the manikin stopped breathing, made the students react as if it was the human body that was failing. The instructors' comments re-directed the students' attunement to the predetermined, agreed scenario.

The enactment of bodily positions in sync also showed that when the medical students performed their tasks, such as palpating the pulse and reporting the rate, the nursing students anticipated the coming action by the medical students and handled them the necessary material equipment such as a torch or an oxygen mask. The interprofessional relationship between the medical and the nursing students was bundled with material entities, present or imagined in the location for the scenario. 


\section{Bodily positionings out of synchrony}

When bodily positionings were out of synchrony with the sociomaterial arrangements, the fluidity of movements became disconnected by task focused performance and dual agendas. Noticing signs of deterioration and taking action did not to lead immediate attunement to the action of the others or responding through anticipation of the next step. Instead, the pattern of movements showed that parallel professional enactments without connection were taking place through the enactment of designated professional actions, as exemplified below by a transcribed video sequence of the nursing and medical students' positioning in the emergency room. However, the chain of actions was eventually connected again by enactment of leadership from either the medical or the nursing students, making dual agendas coalesce.

The nursing students stand in one corner of the room discussing how and when to prepare a urinary catheter. The two medical students stand in another corner of the room, looking for medical dosages in a drug compendium./.../After a while the medical students decide that the patient urgently needs to be transported to the X-ray room. The nursing students stop preparing the urinary catheter and help prepare the patient. (Site $1-$ simulation 2).

This type of interprofessional collaboration is also illustrated graphically in (figure 2) (Insert figure 2 here)

At the beginning of this example, there is parallel professional enactment since there is no collaboration between the two professions in their sayings, doings, or bodily positioning. Then 
the medical students take the lead and the nurse students react to this despite that they cannot complete their planned action. The dual agendas then coalesce. In this example, the students are performing the task assigned to their profession, but in doing so they enact the patients' body in different ways. The medical students are focusing on the medical body and the nursing students on the human body, trying to make the patient as comfortable as possible. The medical students decide that the medical body needs to be prioritised over the human body making the nursing students' preparations of the catheter for the moment unnecessary. Again, this is caused by deficient communication, or sayings, between the two professions.

An example of bodily positioning being out of synchrony is the enactment of task-focused actions where a nursing and a medical student are going to suture a patient who has an wound to his leg, also illustrated graphically in figure 3.

(insert figure 3 here)

... come into the room and there is nothing "alarming" about the patient but the medical student directly starts to go through the procedure of ATLS, declaring that they have a clear airway, the lungs sound good and that they need to get a blood pressure reading. The nursing student tries to prompt the medical student that they need to suture the wound, but the medical student does not listen. The patient says, "Why are you doing all these examinations on me? I'm not sick...". The medical student answers “No, no, we are just examining you and soon we are going to take care of your wound.”. She proceeds with her examinations following the ATLS 
protocol. The nursing student assists when requested, measuring the temperature etc. Otherwise the nursing student remains quiet but starts to prepare for the suture by arranging gloves and aprons, and a tray with syringe, anaesthetic and bandages. When the medical student has completed the examination according to the ATLS protocol she focuses on the wound and asks the patient if he has been anesthetized before. The patient does not really know. The medical student asks the nurse to bring out the adrenaline "If something would happen...”. (Site 2 - simulation 3)

The medical student's doings were all focused on following the protocol in the search problem while the nursing student silently was doing the task given, as well as preparing to take care of the patients' wound. Only brief moments of collaboration between the medical and nursing student was observed.

\section{Discussion}

This paper has explored how nursing and medical students enact interprofessional collaboration in simulated emergency scenarios, applying a practice theory perspective. Practice theory perspectives (Schatzki, 2002; Gherardi, 2009; Green \& Hopwood, 2015; Kemmis, 2009) emphasise professional knowing in practice as emergent, embodied and relational, intertwined with ethical reasoning and materiality. Issues of corporeality, i.e. the significance and role of the body have been put forward as an important dimension of what it means to be, to practice and to learn as a professional, that have implications for professional education (Green \& Hopwood, 2015).

Applying a theoretical framework of practice theory directs the gaze to other dimensions than those that have been most commonly focused on in previous research on interprofessional 
education and simulation (Gough et al., 2012; Palaganas, Epps, \& Reamer, 2014; Alinier et al., 2014). Research on course evaluations and learners' views on IPE, and their learning about the other roles and skills of other professions or disciplines, together with their opinions of taking part in simulation activities are important feedback for educational planning. However, the dominance of evaluative and protocol-driven research as suggested by Berrigan (2011) might preserve taken-for-granted ideas on simulation as an educational strategy and thereby limit the possibilities of development of the pedagogy to better support students' learning. Dieckmann et al. (2012) have argued that theory-driven and process-oriented studies of simulation practices are needed. The findings described in this paper suggest that there is a need to broaden the scope of theories beyond psychology to be able to understanding simulation as a practice. Practice theory can be considered as an alternative theoretical framework that can contribute to an understanding of how interprofessional learning in simulation is entangled with sociomaterial arrangements. This study adds to the existing literature on interprofessional simulation, as it is providing new knowledge on how interprofessional collaboration actually is played out in practice. Previous studies have predominantly focused on participants' opinions of interprofessional simulation as a means of learning interprofessional collaboration, or as part of course evaluative frameworks (e.g. Cook et al., 2011, Gough et al., 2012; Alinier, Harwood, Harwood, Montague, Huish \& Ruparelia, 2014).

The findings of this study can contribute to the development of simulation pedagogy for interprofessional learning with students, through the articulation of concepts that can be used to disentangle the complexity of simulation as an emerging practice. An important finding for these purposes was how the students’ knowing-in-practice was embodied and relational in the 
enactment of the manikin as body in different ways. The students shifted their attunement to the manikin/patients’ body as being a technical, medical or human body. These shifts demonstrate how students' actions and interactions within the simulation are entangled with material arrangements. One plausible interpretation is also that the shifting attunements to different enactments of a medical or human body encompass possible fragments of professional perspectives of doctors and nurses in play. Students' attunement to the manikin/patient as a technical body was intentionally related to the material arrangements of the simulation that required the participants to perform certain activities in ways that the technology allowed. However, the findings also demonstrate that the breakdown of the technical body, lead the students to intentionally relate to the manikin as being a medical and human body. However, recent theorisations of practice (Kinsella, 2015) have suggested that a focus on the role of the body in professional practices, in simulated or naturalistic settings, might enable educators and learners to draw attention to other dimensions of knowledge, that are not easily accessible through cognitive perspectives, 'dimensions that might help us illuminate, understand and investigate other types of knowledge that are relevant to everyday practices’ (p. 294). Recognising the role of the body in knowledge production in practice goes beyond a focus on the individual practitioner, in the clarification how the performance of a practice is constituted by the relational nature of material arrangements and professional bodies (Kinsella, 2015). The second key findings in this article was that the interprofessional collaboration in the simulation room was enacted as bodily positionings in and out of synchronisation in a fluid way. Students' bodily positionings emerged in relation to the sociomaterial entities as well as the arrangement of the simulation room. When in sync, the students performed and enacted interprofessional collaboration where saying, doings and bodily movements were connected in a 
chain of actions. Typically, when chains of actions were connected, and in sync, interprofessional collaboration emerged through the way they enacted their respective professional knowing in the context of others', participants were attuned to leading or responding to others' sayings and doings. When chains of actions were out of sync, the bodily movements were disconnected, creating parallel professional enactments. These findings could be related to what Johnson (2015), in a dynamic perspective on practice and practicing bodies, has described as 'enacted and embodied rhythms to practice' that generate a periodicity that enable practitioner to construct their practice together. There are multiple rhythms of various kinds in a practice, and these help the practitioners to sort the choices of actions undertaken. Johnson (2015) suggests that the sensitive synchronisation of practitioners' bodily actions and understandings is what shapes their professional practice.

The practice theory perspective, and observational methodology chosen in the research project, provided tools that brought about new understandings of interprofessional collaboration, through disentangling the sociomaterial relationships in simulation as a practice setting. Data was generated from two different universities and created a common pool of data for analysis, which can contribute to increase the transferability across learning settings. The scenarios involved all concerned interprofessional collaboration in simulated acute emergency settings, but the findings of being in and out of synchrony in collaboration might be transferable to interprofessional collaboration in general. A critical reflection is that the methodology was delimited to what could be captured through observations and documents. Additional interviews with participants after simulation might have enriched the understanding even further. However, the process of collective 
data analysis enriched the interpretations made. The iteration and balance between empirical data and theory required a constant critical reflection and a negotiated consensus on identified themes in the analysis.

\section{Concluding remarks}

The practice theory perspective applied and articulation of different enactments of the patient's

body as medical, human and technical, could contribute to the renewal of simulation pedagogy. The concepts of interprofessional collaboration as bodily positionings in and out of synchrony can be seen as what Kinsella (2015) describes as reflecting a view of mind and body as integrated, the mind is reflected in the bodily moves, actions and activities of the participants. The articulation of bodily rhythms in interprofessional simulation practice as in or out of synchrony can be used analytically in simulation activities in order to support and improve participants’ learning in new ways.

\section{Acknowledgement}

The authors wish to thank the Swedish Research Council for funding of the SIMIPL project, and the partners at Göteborg University and the Karolinska Institutet for collecting and sharing the data analysed in this paper. We would also like to acknowledge Dr Nick Hopwood, University of Technology, Sydney, for acting as a critical friend, particularly for his input to the analysis and graphical presentation of our findings.

\section{Declaration of interest}

The authors report no declarations of interest. 


\section{References}

Ahn, S-E., Rimpiläinen, S., Theodorsson, A., Fenwick, T. \& Abrandt Dahlgren, M. (2015).

Learning in technology enhanced medical simulation: Locations and knowings. Professions and Professionalism, 5(1), 1-12.

Alinier, G., Harwood, C., Harwood, P., Montague, S., Huish, E., Ruparelia, K., \& Antuofermo, M. (2014). Immersive Clinical Simulation in Undergraduate Health Care Interprofessional Education: Knowledge and Perceptions. Clinical Simulation In Nursing, 10(4), e205-16

Berragan, L. (2011). Simulation: an effective pedagogical approach for nursing? Nurse Education Today, 31(7), 660-663.

Boeije, H. (2002). A Purposeful Approach to the Constant Comparative Method in the Analysis of Qualitative Interviews. Quality \& Quantity, 36: 391-409

Cant, R. P., \& Cooper, S. J. (2010). Simulation-based learning in nurse education: systematic review. Journal of Advanced Nursing, 66(1), 3-15

Cook et al., (2011). Technology-enhanced simulation for health professions education: A systematic review and meta-analysis. JAMA, 7, 306(9), 978-988

Dieckmann, P., Molin Friis, S., Lippert, A., \& Østergaard, D. (2012). Goals, success factors, and barriers for simulation-based learning: a qualitative interview study in health care. Simulation \& Gaming, 43(5), 627-647. 
Fenwick, T., \& Dahlgren, M. A. (2015). Towards sociomaterial approaches in simulation-based education: lessons from complexity theory. Medical Education, 49(4), 359-367.

Fenwick, T. (2010). Re-thinking the "thing." Socio material approaches to understanding and researching learning in work. Journal of workplace learning, 22(1/2), 104-116

Frenk J. et al. (2010). Health professional for a new century: Transforming education to strengthen health systems in an interdependent world. Lancet, 376, 9756.

Gherardi, S. (2009) Knowing and learning in practice-based studies: an introduction. The Learning Organization, 16(5), 352-359

Gough, S., Hellaby, M., Jones, N. \& MacKinnon, R. (2012). A review of undergraduate interprofessional simulation-based education. Collegian, 19(3), 153-171

Green, B., \& Hopwood, N. (Eds.) The Body in Professional Practice, Learning and Education: Body/Practice. Dordrecht: Springer.

\section{Fenwick, T., Edwards, R. and Sawchuk, P. (2011), Emerging Approaches to Educational Research: Tracing the Sociomaterial, Routledge, London.}

Hager, P., Lee, A. \& Reich, A. (2012) (Eds) Practice, learning and change. Practice theory perspectives on professional learning. Dordrecht: Springer 


\section{Hammersley, M. (2006). Ethnography: problems and prospects. Ethnography and}

Education, 1(1), 3-14.

Heath, C., Hindmarsh, J, \& Luff, P. (2010). Video in qualitative research. Analysing Social interaction in Everyday Life. Los Angeles: SAGE.

Hopwood, N., Kelly, M., Boud, D. \& Rooney, D. (2014). Simulation in Higher Education: A sociomaterial view. Education philosophy and theory. DOI:10.1080/00131857.2014.971403

Hopwood, N. (2013). Using video to trace the embodied and material in a study of health practice. Qualitative research journal. 14(2), 1997-211.

Johnson, M.C. (2015) Terroir and timespace: Body rhythms in winemaking. In: B. Green \& N. Hopwood, (Eds.) The Body in Professional Practice, Learning and Education: Body/Practice (pp. 71-88). Dordrecht: Springer.

Kemmis, S. (2009). Understanding professional practice: A synoptic framework. In B. Green (Ed.) Understanding and researching professional practice (pp. 19-38). Rotterdam: Sense Publishers.

Kinsella, E. A. (2015) Embodied knowledge: Towards a corporeal turn in professional practice, research and education. In: B. Green \& N. Hopwood, (Eds.) The Body in Professional Practice, Learning and Education: Body/Practice (pp. 245-261). Dordrecht: Springer. 
Palaganas, J. C., Epps, C., \& Reamer, D. (2014). A history of simulation-enhanced interprofessional education. Journal of Interprofessional Care, 28(2), 110-115.

Schatzki, T. (2002). The site of the social: A philosophical account of the constitution of social life and change. University Park: Pennsylvania State University Press.

Schatzki, T.R. (2012). A primer on practices , in Higgs, J., Barnett, R., Billett, S., Hutchings, M. and Trede, F. (Eds), Practice-Based Education: Perspectives and Strategies, Sense, Rotterdam, pp. 13-26.

World Health Organization (2010). Framework for action on interprofessional Education and Collaborative Practice. WHO; Geneva. 
(To be inserted at page: 7, line 151)

Table 1: Phases in the analytical process

\begin{tabular}{|c|c|c|c|c|}
\hline $\begin{array}{l}\text { Type of } \\
\text { comparison (phase) }\end{array}$ & Purpose & Analysis activities & Questions & Results \\
\hline $\begin{array}{l}\text { 1. Comparison of } \\
\text { individual field notes } \\
\text { from a single video- } \\
\text { recording }\end{array}$ & $\begin{array}{l}\text { Develop } \\
\text { collectively } \\
\text { enriched and } \\
\text { shared } \\
\text { understanding of } \\
\text { the sequence of } \\
\text { activities }\end{array}$ & $\begin{array}{l}\text { Merging individual } \\
\text { field notes, reaching } \\
\text { consensus on } \\
\text { interpretations of } \\
\text { fragments }\end{array}$ & $\begin{array}{l}\text { How is inter- } \\
\text { professional colla- } \\
\text { boration enacted? } \\
\text { How are different } \\
\text { professional knowings } \\
\text { made relevant? } \\
\text { How are socio- } \\
\text { material arrangements } \\
\text { related to sayings and } \\
\text { doings? }\end{array}$ & $\begin{array}{l}\text { Focus and process } \\
\text { for phase } 2 \\
\text { determined } \\
\text { Structural features } \\
\text { for comparing video } \\
\text { recordings } \\
\text { formulated }\end{array}$ \\
\hline $\begin{array}{l}\text { 2. Comparison } \\
\text { between different } \\
\text { video recordings of } \\
\text { the same scenario }\end{array}$ & $\begin{array}{l}\text { Develop a shared } \\
\text { understanding of } \\
\text { the patterns } \\
\text { across the data of } \\
\text { each phase of the } \\
\text { sequence }\end{array}$ & $\begin{array}{l}\text { Comparing observed } \\
\text { interconnections } \\
\text { between human and } \\
\text { non-human actors }\end{array}$ & $\begin{array}{l}\text { How is the sequence of } \\
\text { activities in the } \\
\text { simulation enacted } \\
\text { and related to socio- } \\
\text { material } \\
\text { arrangements? }\end{array}$ & $\begin{array}{l}\text { Transcripts of } \\
\text { selected segments } \\
\text { Preliminary patterns } \\
\text { of interprofessional } \\
\text { collaboration as a } \\
\text { focus for phase } 3\end{array}$ \\
\hline $\begin{array}{l}\text { 3. Comparison } \\
\text { between video } \\
\text { recordings of } \\
\text { different scenarios }\end{array}$ & $\begin{array}{l}\text { Enlarging and } \\
\text { enriching the } \\
\text { basis for } \\
\text { interpretation } \\
\text { Identifying } \\
\text { variation }\end{array}$ & $\begin{array}{l}\text { Refining provisional } \\
\text { interpretations against } \\
\text { wider data }\end{array}$ & $\begin{array}{l}\text { How is } \\
\text { interprofessional } \\
\text { collaboration enacted } \\
\text { and stabilised across } \\
\text { scenarios? }\end{array}$ & $\begin{array}{l}\text { Relational patterns } \\
\text { of enacted } \\
\text { interprofessional } \\
\text { collaboration across } \\
\text { all data }\end{array}$ \\
\hline
\end{tabular}


To be inserted at page: 9 (line 198)

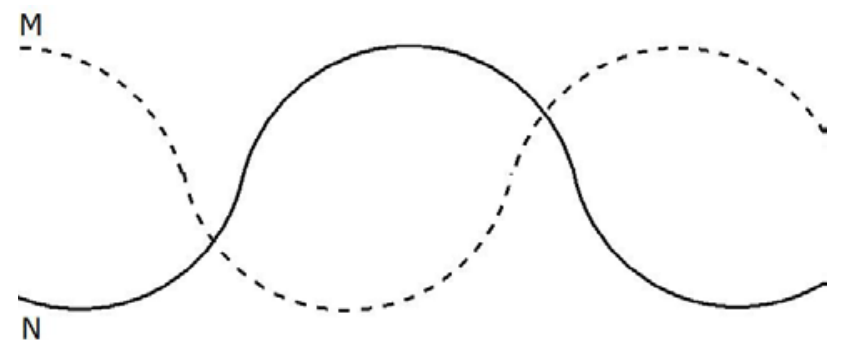

Figure 1: Interprofessional collaboration between nursing (N) and medical students (M) as bodily positionings in synchrony with socio-material arrangements.

To be inserted at page: 11 (line 248)

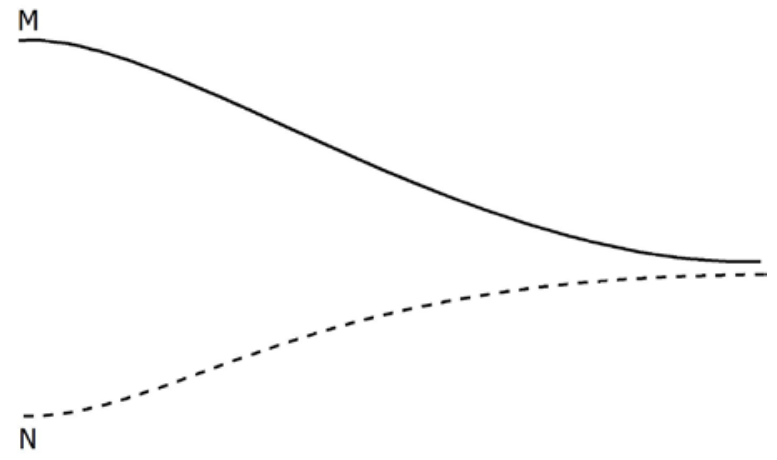

Figure 2: Interprofessional collaboration between nursing (N) and medical students (M) as bodily positionings out of synchrony with socio-material arrangements. Dual agendas coalesce.

To be inserted at page: 12 (line 264)

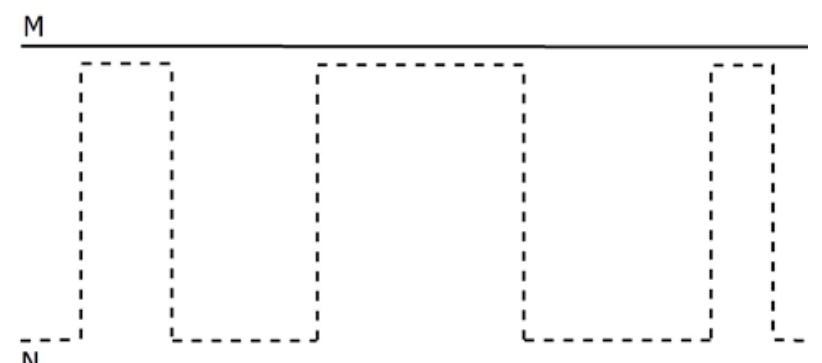

Figure 3: Interprofessional collaboration between nursing $(\mathrm{N})$ and medical students $(\mathrm{M})$ as bodily positionings out of synchrony with socio-material arrangements. Both professional groups have a task focus. 